\title{
Factores relacionados con la empatía en estudiantes de medicina de la Universidad de Cartagena
}

\author{
Meisser Vidal Madera Anaya ${ }^{a}$, Lesbia Rosa Tirado Amador ${ }^{b}$ y Farith González Martínez ${ }^{c}$
}

aOdontólogo, Universidad de Cartagena. Magíster en Epidemiologia Clínica, Universidad de La Frontera. Magíster en Bioquímica (c), Universidad de Cartagena.

'Odontóloga, Universidad de Cartagena. Investigador asociado al grupo de Salud Pública (GISPOUC).

'Odontólogo. Magíster en Salud Pública. Profesor asociado. Facultad de Odontología Universidad de Cartagena.

\section{Correspondencia:}

Meisser Vidal Madera Anaya. Universidad de La Frontera; Centro de Capacitación, Investigación y Gestión para la Salud Basada en la Evidencia CIGES; Facultad de Medicina. Temuco-Chile.

Correo:

meissermadera@gmail.com

Recibido el 24 de junio de 2015.

Aceptado para su publicación el 20 de agosto de 2015.
Objetivo: Determinar los niveles de empatía y su relación con factores sociodemográficos, familiares y académicos en estudiantes de Medicina.

Diseño: Estudio de corte transversal.

Emplazamiento: Universidad de Cartagena (Colombia).

Participantes: 256 estudiantes de Medicina de la Universidad de Cartagena, seleccionados aleatoriamente.

Mediciones Principales: Se aplicó un cuestionario que indagaba sobre características sociodemográficas, familiares, académicas y la Escala de Empatía Médica de Jefferson versión S. Para el análisis se utilizó la prueba de Shapiro-Wilk para evaluar el supuesto de normalidad, las pruebas T Student y Anova para establecer la relación entre variables $(p<0,05)$.

Resultados: La media de empatía global fue $114,3 \pm 12,8$ puntos. Se encontraron diferencias estadísticamente significativas entre la media de empatía global con el sexo $(p=0,0033)$, funcionalidad familiar $(p=0,0017)$, Medicina como primera opción de estudio $(p=0,03)$, rendimiento académico $(p=0,0464)$ y promedio académico acumulado $(p=0,003 ; r=0,19)$.

Conclusiones: Los niveles de empatía en estudiantes de Medicina pueden variar dependiendo del sexo, funcionalidad familiar, primera opción de estudio, promedio y rendimiento académico; lo que hace imperativa la implementación de estrategias pedagógicas en las áreas humanas involucrando a las familias de los educandos, mejorando así los niveles de empatía y la atención en salud.

PALABRAS CLAVE: Empatía. Estudiantes de Medicina. Relaciones Médico-Paciente.

\section{ABSTRACT}

Factors related to empathy in medical students at the University of Cartagena

Objective: To determine empathy levels and its relationship with sociodemographic, academic and family factors in medical students.

Design: Cross-sectional study.

Location: University of Cartagena (Colombia).

Participants: 256 medical students at the University of Cartagena, randomly selected.

Main measures: A questionnaire was applied that asked about sociodemographic, family and academic factors and the Jefferson Scale of Physician Empathy version S. Shapiro-Wilk test was used to assess the normality assumption, and T Student and Anova tests were used to establish relationship among variables $(p<0.05)$.

Results: The average global empathy was $114.3 \pm 12.8$ points; we found statistically significant differences between the average global empathy with sex $(p=0.0033)$, family functioning $(p=0.0017)$, Medicine as first choice study $(p=0.03)$, academic performance $(p=0.0464)$ and cumulative grade point average $(p=0.003 ; r=0.19)$.

Conclusions: The levels of empathy in medical students may vary depending on gender, family functioning, first choice of study, academic average and performance; which makes it imperative to implement pedagogical strategies in human areas involving students' families, and therefore improving the levels of empathy and health care.

KEY WORDS: Empathy. Medical Students. Physician-Patient Relations. 


\section{INTRODUCCIÓN}

La prestación de servicios de salud se enfoca en la satisfacción de las necesidades de los pacientes en todas sus dimensiones, las cuales pueden encontrarse influenciadas por percepciones, sentimientos y emociones. El reconocimiento de estos factores emocionales y psicosociales es uno de los retos que afronta el profesional de la atención primaria, pudiéndose lograr por medio del desarrollo de la empatía, una habilidad o atributo cognitivo que tiene efecto positivo en la relación médico-paciente ${ }^{1}$. Esta es considerada un elemento esencial de la personalidad para alcanzar la competencia clínica y el profesionalismo en Medicina ${ }^{2}$, ya que facilita la comunicación y la comprensión, hecho que genera confianza por parte del paciente y permite mejorar el quehacer profesional en aspectos básicos de la atención primaria como anamnesis, exámenes físicos, diagnósticos y tratamientos ${ }^{3}$.

Una atención cercana a la realidad de los pacientes influirá en su grado de satisfacción y motivación para que asuman actitudes participativas y estables en sus planes de tratamiento ${ }^{4,5}$, por lo cual el desarrollo de empatía en los estudiantes de Medicina se ha convertido en una necesidad y preocupación de las instituciones educativas ${ }^{6}$, a tal nivel que consideran que debe ser abordada específicamente en el plan de estudio básico junto con habilidades de comunicación ${ }^{7}$. Por esto, durante algunos años se han desarrollado diversos instrumentos estandarizados para evaluar los niveles de empatía ${ }^{8}$, siendo uno de los más utilizados la Escala de Empatía Médica de Jefferson (EEMJ) ${ }^{9}$, diseñada inicialmente para evaluar la empatía en Médicos y modificada para poder ser aplicada en estudiantes de Medicina y de otras áreas de atención en salud ${ }^{10}$.

Los niveles de empatía no son estáticos, por lo que algunos factores pueden modificarlos; en este sentido es posible mencionar que existen algunos estudios en Europa ${ }^{7}$, Asia ${ }^{11}$ y América Latina ${ }^{12,13}$ con enfoque en evaluar la validez y confiabilidad de la EEMJ y, a su vez, los niveles de empatía en virtud de factores como la edad, sexo y año académico. Sin embargo existen pocos reportes de niveles de empatía en relación a los factores de tipo familiar ${ }^{14}$.

El objetivo de este estudio fue determinar los niveles de empatía y su relación con factores sociodemográficos, familiares y académicos en estudiantes de Medicina de la Universidad de Cartagena durante el segundo periodo académico del año 2014.

\section{MATERIAL Y MÉTODOS}

Estudio de corte transversal realizado en estudiantes de Medicina de la Universidad de Cartagena. Se seleccionaron aleatoriamente 256 participantes y la muestra fue calculada teniendo en cuenta un nivel de confianza del $95 \%$, precisión del $1 \%$ y desviación típica de 15,115. Este estudio fue aprobado por el comité de ética de la Universidad de

\section{Cartagena.}

Para la inclusión de los participantes se tuvieron en cuenta a estudiantes regulares del programa de Medicina matriculados en el segundo periodo académico del 2014, que aceptaron y firmaron el consentimiento informado. Se excluyeron a estudiantes de internado y postgrados, además a estudiantes de otras universidades que al momento de la medición estuvieran realizando pasantías, intercambios o prácticas en la Universidad de Cartagena.

\section{Instrumentos y mediciones}

Cuestionario estructurado. Permitió obtener información sobre las características sociodemográficas, académicas y familiares: edad, sexo, estado civil, estrato socioeconómico, procedencia, trabajo y estudio, año de formación, Medicina como primera opción de estudio, reprobación de asignaturas, reprobación de la misma asignatura más de una vez, estudio previo de otra profesión y tipo de familia (anexo).

APGAR familiar. Muestra cómo perciben los miembros de la familia el nivel de funcionamiento de la unidad familiar ${ }^{16}$. Al sumar las cinco respuestas el puntaje varia de 0 a 20 puntos, estableciendo cuatro categorías: función normal (18-20 puntos), disfunción leve (13-17 puntos), disfunción moderada (10-12 puntos) y disfunción severa ( $\leq 9$ puntos). Contiene 2 preguntas para evaluar el soporte de amigos, el punto de corte es $\leq 4$ sin soporte y $>$ 4 con soporte. Este instrumento ha sido validado reportando una consistencia interna de 0,7917-20.

Escala de Empatía Médica de Jefferson (EEMJ). La versión en español para estudiantes de Medicina validada en México ${ }^{12}$ tiene una estructura consistente con los aspectos conceptuales de una escala multidimensional| ${ }^{21-23}$, que define a la empatía en base a tres factores: "toma de perspectiva", "cuidado con compasión" y la habilidad para "ponerse en el lugar del paciente". Tiene una puntuación mínima de 20 , máxima de 140 y no establece punto de corte, contiene 20 ítems tipo Likert en una escala de 7 puntos. Existen 10 ítems redactados en sentido positivo y 10 en sentido negativo; tiene una consistencia interna de $0,89^{24}$.

Se realizó una prueba piloto con 30 individuos de características similares. En el instrumento tipo cuestionario se evaluó la comprensión de cada una de las preguntas, la suficiencia de las categorías y la extensión y pertinencia de las mismas, lo que permitió hacer las modificaciones necesarias para que pudiera ser aplicado, de este modo se adecuó el cuestionario en torno al contexto y se validó en apariencia a partir de dos jueces expertos.

Se seleccionaron los participantes por medio de un programa de números aleatorios, se contactaron e invitaron a participar en el estudio, explicándoles los objetivos, beneficios y riesgos; todos los participantes debían inicialmente autorizar su partici- 
pación a través de la firma de su consentimiento informado por escrito. Los cuestionarios fueron suministrados en un solo momento y en una única sala teniendo en cuenta el año de formación. El proceso de aplicación estuvo a cargo de dos operadores neutrales, resguardando la confidencialidad.

Luego, con previa autorización expresada en el consentimiento informado, se solicitó a la oficina de admisiones y registro de control académico de la Universidad de Cartagena el promedio académico acumulado de todos los estudiantes participantes. Esta información se utilizó para determinar la variable promedio académico y rendimiento académico.

\section{Análisis estadístico}

Se utilizaron pruebas de estadística descriptiva (promedios, desviación estándar, distribución de frecuencias y porcentajes). Para la escala de empatía y sus dimensiones los datos fueron sometidos a la prueba de normalidad de Shapiro-Wilk. Luego se evaluó la consistencia interna del instru- mento a partir de la prueba alfa de Cronbach; la comparación entre los grupos se realizó mediante las pruebas T Student y Anova. Para evaluar la relación entre la variable empatía y promedio académico se realizó una regresión lineal simple y se utilizó el índice correlación de Pearson. Adicionalmente para evaluar la asociación entre empatía con más de una variable se diseñaron modelos de asociación a través de regresión lineal múltiple, luego se validaron los modelos a través de la evaluación de los supuestos mediante gráficos de residuos y el test para datos influyentes distancia de Cook. Para todos estos análisis se asumió un límite de significancia de 0,05 y se usó el programa estadístico STATA® (Stata Corp. LP, College Station, TX, USA) versión 12.0 .

\section{RESULTADOS}

El promedio de edad de todos los participantes fue de 19,5 (DE: 2,3) años; las características sociodemográficas se muestran en la tabla 1. El valor de confiabilidad y estabilidad de la escala de EEMJ versión $\mathrm{S}$ tomado con la prueba alfa de Cronbach fue de 0,79 .

\begin{tabular}{|c|c|c|}
\hline Características sociodemográficas & $\mathbf{N}$ & PORCENTAJE (\%) \\
\hline \multicolumn{3}{|l|}{ Sexo } \\
\hline - Masculino & 118 & 46,1 \\
\hline - Femenino & 138 & 53,9 \\
\hline \multicolumn{3}{|l|}{ Edad según ciclo vital } \\
\hline - Adolescente & 112 & 43,7 \\
\hline - Adulto joven & 144 & 56,3 \\
\hline \multicolumn{3}{|l|}{ Estado civil } \\
\hline - Soltero & 252 & 98,4 \\
\hline - Casado & 2 & 0,8 \\
\hline - Unión libre & 2 & 0,8 \\
\hline \multicolumn{3}{|l|}{ Religión } \\
\hline - Católico & 183 & 71,5 \\
\hline - Evangélico & 36 & 14,1 \\
\hline - Otras/ninguna & 37 & 14,4 \\
\hline \multicolumn{3}{|l|}{ Procedencia } \\
\hline - Urbana & 207 & 80,9 \\
\hline - Rural & 49 & 19,1 \\
\hline \multicolumn{3}{|l|}{ Estrato socioeconómico } \\
\hline - Muy bajo & 37 & 14,4 \\
\hline - Bajo & 70 & 27,3 \\
\hline - Medio Bajo & 90 & 35,2 \\
\hline - Medio & 47 & 18,4 \\
\hline - Medio alto & 10 & 3,9 \\
\hline - Alto & 2 & 0,8 \\
\hline Total & 196 & 100 \\
\hline
\end{tabular}

Tabla 1. Características sociodemográficas de los participantes 
Con relación a las características familiares, el $60,9 \%$ pertenecían a una familia nuclear, el $60,5 \%$ a familias en disfuncionalidad y el 91,2 \% contaban con el soporte de amigos. Respecto a las características académicas, el 89,0\% estaban estudiando la profesión que tenían como primera opción, el $9,8 \%$ trabajaban y estudiaban simultáneamente, el $28,5 \%$ habían reprobado alguna vez al menos una asignatura y el 3,1\% había reprobado más de una vez la misma asignatura, el 15,2\% había estudiado una profesión previa y el $51,6 \%$ tenían un rendimiento académico bueno. La media del promedio académico acumulado de los estudiantes participantes fue de 3,71 (DE: 0,34$)$ puntos, con un valor mínimo de 2,78 puntos y máximo de 4,52 puntos.

La media de empatía global de los participantes fue de 114,3 (DE: 12,8) puntos, según sus dimensiones la media de la "toma de perspectiva" fue de 61,4 (DE: 6,6) puntos, para el "cuidado con compasión" de 41,7 (DE: 7,8) puntos y "ponerse en el lugar del paciente" de 11,2 (DE: 3,2) puntos. Al comparar los niveles de empatía global y sus dimensiones con las características sociodemográficas se encontraron diferencias estadísticamente significativas con el sexo en la empatía global $(p=0,0033)$ y en la dimensión "cuidado con compasión" $(p=0,001)$, en donde las mujeres reportaron mayores niveles de empatía (figura 1).

Respecto a los niveles de empatía y sus dimensiones con las características familiares se encontraron diferencias estadísticamente significativas con la funcionalidad familiar en los niveles de empatía

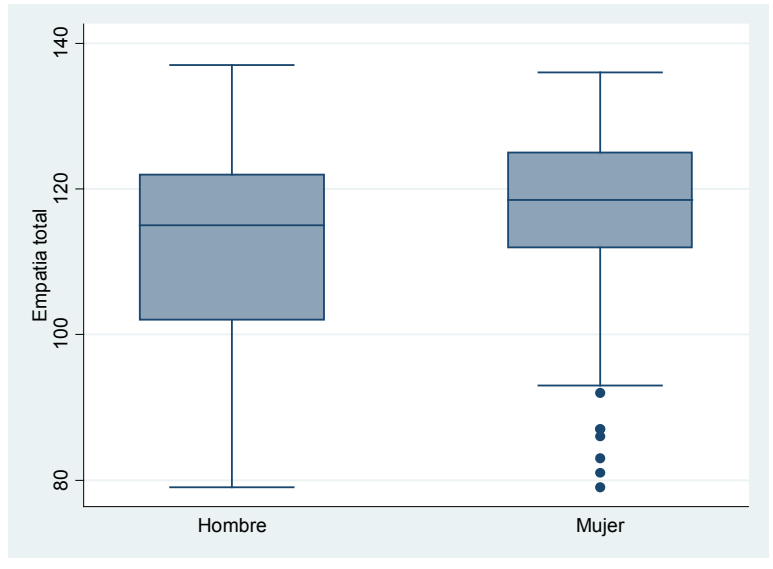

Figura 1. Relación entre la empatía global y el sexo en estudiantes de Medicina de la Universidad de Cartagena. *Prueba T Student: $p=0,0033$.

global $(p=0,0017)$ y en la dimensión "toma de perspectiva" ( $p=0,0005)$ (tabla 2$)$.

Al relacionar los niveles de empatía y sus dimensiones con las características académicas, se encontraron diferencias significativas entre la media de empatía global y Medicina como primera opción de estudio $(\mathrm{p}=0,03)$; del mismo modo entre la dimensión "ponerse en el lugar del paciente" y Medicina como primera opción de estudio $(p=0,0004)$; asimismo entre la dimensión "toma de perspectiva" y el rendimiento académico $(p=0,0464)$ (tabla 3). La

\begin{tabular}{|c|c|c|c|c|c|c|c|c|}
\hline \multicolumn{9}{|c|}{ Empatía y factores familiares } \\
\hline & \multicolumn{2}{|c|}{ Toma de perspectiva } & \multicolumn{2}{|c|}{$\begin{array}{l}\text { Cuidado con } \\
\text { compasión }\end{array}$} & \multicolumn{2}{|c|}{$\begin{array}{l}\text { Ponerse en el lugar } \\
\text { del paciente }\end{array}$} & \multicolumn{2}{|c|}{ Empatía global } \\
\hline & $M \pm D E$ & $\mathbf{p}$ & $M \pm D E$ & $\mathbf{p}$ & $M \pm D E$ & $\mathbf{p}$ & $M \pm D E$ & $\mathbf{p}$ \\
\hline \multicolumn{9}{|l|}{ Tipo de Familia ${ }^{\varepsilon}$} \\
\hline Nuclear & $61,5 \pm 6,6$ & 0,55 & $41,4 \pm 7,9$ & 0,39 & $11,2 \pm 3,3$ & 0,94 & $114,0 \pm 13,0$ & 0,42 \\
\hline Extensa & $59,9 \pm 7,4$ & & $40,8 \pm 6,8$ & & $11,1 \pm 3,3$ & & $111,7 \pm 12,5$ & \\
\hline Nuclear ampliada & $62,0 \pm 6,3$ & & $43,0 \pm 9,4$ & & $11,2 \pm 3,2$ & & $116,2 \pm 15,0$ & \\
\hline Binuclear & $63,5 \pm 6,5$ & & $46,1 \pm 2,3$ & & $10,5 \pm 2,1$ & & $120,1 \pm 5,1$ & \\
\hline Monoparental & $61,5 \pm 6,0$ & & $42,3 \pm 7,7$ & & $11,5 \pm 3,1$ & & $115,3 \pm 11,5$ & \\
\hline \multicolumn{9}{|l|}{ Función familiar ${ }^{\dagger}$} \\
\hline Disfuncional & $60,3 \pm 6,8$ & $0,0005^{\star}$ & & 0,07 & $11,0 \pm 3,1$ & 0,11 & $112,4 \pm 13,0$ & $0,0017^{\star}$ \\
\hline Funcional & $63,1 \pm 6,0$ & & & & $11,5 \pm 3,3$ & & $117,1 \pm 12,0$ & \\
\hline \multicolumn{9}{|c|}{ Soporte de amigos ${ }^{\dagger}$} \\
\hline $\mathrm{Si}$ & $61,5 \pm 6,6$ & 0,22 & & 0,08 & $11,2 \pm 3,2$ & 0,34 & $114,6 \pm 12,7$ & 0,08 \\
\hline No & $60,3 \pm 6,4$ & & & & $10,9 \pm 2,9$ & & $110,7 \pm 13,4$ & \\
\hline
\end{tabular}

Tabla 2. Relación entre la empatía global y sus dimensiones con las características familiares de los participantes. Datos en media (M) y desviación estándar (DE); *Estadísticamente significativo p<0,05; 'Prueba T Student; ' $P$ Prueba Anova. 
relación entre la media de empatía global y el promedio académico fue estadísticamente significativa $(p=0,003, r=0,19)$.

Al realizar el análisis de regresión lineal múltiple en donde se utilizó la empatía como variable respuesta, la funcionalidad familiar como exposición y las variables sexo y rendimiento académico como controles, la funcionalidad familiar se asoció significativamente a los niveles de empatía $(p=0,000)$, controlando por sexo y rendimiento académico. Este modelo de asociación solo explica el 12,1\% de la variabilidad existente en los datos. También sugiere que en los estudiantes de Medicina se espera con un $95 \%$ de confianza que el incremento en los niveles de empatía sea entre 2,19 y 7,57 puntos, dependiendo de la funcionalidad familiar para un sexo y rendimiento académico fijo (tabla 4).

Empatía y factores familiares

\begin{tabular}{|c|c|c|c|c|c|c|c|c|}
\hline & \multicolumn{2}{|c|}{ Toma de perspectiva } & \multicolumn{2}{|c|}{$\begin{array}{l}\text { Cuidado con } \\
\text { compasión }\end{array}$} & \multicolumn{2}{|c|}{$\begin{array}{c}\text { Ponerse en el lugar } \\
\text { del paciente }\end{array}$} & \multicolumn{2}{|c|}{ Empatía global } \\
\hline & $M \pm D E$ & $\mathbf{p}$ & $M \pm D E$ & $\mathbf{p}$ & $M \pm D E$ & $\mathbf{p}$ & $M \pm D E$ & $\mathbf{p}$ \\
\hline \multicolumn{9}{|c|}{ Año de formación ${ }^{\varepsilon}$} \\
\hline Primero & $62,0 \pm 6,9$ & 0,56 & $41,2 \pm 8,0$ & 0,91 & $11,8 \pm 3,3$ & 0,14 & $114,1 \pm 13,5$ & 0,81 \\
\hline Segundo & $61,0 \pm 6,0$ & & $41,5 \pm 8,4$ & & $10,9 \pm 3,1$ & & $113,4 \pm 11,9$ & \\
\hline Tercero & $60,3 \pm 6,9$ & & $41,9 \pm 8,3$ & & $11,3 \pm 2,7$ & & $113,5 \pm 13,7$ & \\
\hline Cuarto & $62,3 \pm 6,6$ & & $41,6 \pm 6,9$ & & $12,4 \pm 3,8$ & & $116,3 \pm 11,8$ & \\
\hline Quinto & $61,1 \pm 6,6$ & & $42,8 \pm 6,2$ & & $11,3 \pm 3,1$ & & $115,2 \pm 12,6$ & \\
\hline \multicolumn{9}{|c|}{$\begin{array}{l}\text { Medicina como primera } \\
\text { opción }^{+}\end{array}$} \\
\hline Sí & $61,4 \pm 6,7$ & 0,42 & $41,9 \pm 7,8$ & 0,06 & $11,4 \pm 3,1$ & $0,0004^{*}$ & $114,8 \pm 12,8$ & $0,03^{*}$ \\
\hline No & $61,1 \pm 5,5$ & & $39,6 \pm 7,2$ & & $9,3 \pm 3,3$ & & $110,0 \pm 12,1$ & \\
\hline \multicolumn{9}{|c|}{ Estudia y trabaja } \\
\hline $\mathrm{Si}$ & $61,7 \pm 6,4$ & 0,79 & $43,2 \pm 7,0$ & 0,31 & $11,0 \pm 3,1$ & 0,77 & $115,9 \pm 13,1$ & 0,49 \\
\hline No & $61,3 \pm 6,6$ & & $41,5 \pm 7,8$ & & $11,2 \pm 3,2$ & & $114,1 \pm 12,7$ & \\
\hline \multicolumn{9}{|c|}{ Reprobación de asignaturas } \\
\hline Sí & $61,2 \pm 6,6$ & 0,40 & $40,4 \pm 8,5$ & 0,051 & $11,4 \pm 2,9$ & 0,77 & $113,5 \pm 12.9$ & 0,17 \\
\hline No & $61,4 \pm 6,6$ & & $42,2 \pm 7,4$ & & $11,1 \pm 3,3$ & & $114,2 \pm 12,8$ & \\
\hline \multicolumn{9}{|c|}{ Estudio de profesión previa } \\
\hline Sí & $62,1 \pm 5,9$ & 0,43 & $41,6 \pm 8,5$ & 0,92 & $10,8 \pm 3,4$ & 0,42 & $114,5 \pm 12,9$ & 0,88 \\
\hline No & $61,2 \pm 6,7$ & & $41,7 \pm 7,6$ & & $11,2 \pm 3,2$ & & $114,2 \pm 12,8$ & \\
\hline \multicolumn{9}{|c|}{ Rendimiento académico ${ }^{\mathfrak{}}$} \\
\hline Deficiente & $55,7 \pm 8,4$ & $0,0464^{*}$ & $39,0 \pm 7,4$ & 0,20 & $11,4 \pm 3,8$ & 0,64 & $106,1 \pm 11,7$ & 0,08 \\
\hline Regular & $61,2 \pm 6,1$ & & $39,9 \pm 7,9$ & & $10,9 \pm 3,5$ & & $111,9 \pm 12.6$ & \\
\hline Bueno & $61,9 \pm 6,6$ & & $41,7 \pm 8,1$ & & $11,4 \pm 3,2$ & & $115,1 \pm 13,1$ & \\
\hline Muy bueno & $60,8 \pm 6,4$ & & $42,8 \pm 7,0$ & & $10,9 \pm 3,0$ & & $114,5 \pm 12,0$ & \\
\hline Excelente & $67,3 \pm 4,6$ & & $46,7 \pm 4,0$ & & $13,0 \pm 2,0$ & & $127,0 \pm 6,2$ & \\
\hline
\end{tabular}

Tabla 3. Relación entre la empatía global y sus dimensiones con las características académicas de los participantes. Datos en media y desviación estándar; 'Estadísticamente significativo $p<0,05$; 'Prueba T Student; EPrueba Anova. 


\begin{tabular}{|c|c|c|c|c|}
\hline Variables & Beta estimado & Error estándar & $\mathbf{t}$ & Valor $\mathrm{p}$ \\
\hline Intersección & 107,18 & 1,75 & 61,28 & $0,000^{\star}$ \\
\hline Funcionalidad familiar & 4,88 & 1,36 & 3,57 & $0,000^{\star}$ \\
\hline Sexo & 5,12 & 1,34 & 3,81 & $0,000^{\star}$ \\
\hline Rendimiento académico & 4,36 & 1,75 & 2,49 & $0,013^{*}$ \\
\hline
\end{tabular}

Tabla 4. Asociación entre la empatía y la funcionalidad familiar controlados por sexo y rendimiento académico en estudiantes de Medicina de la Universidad de Cartagena. $R$ cuadrado = 0,1323 ( $\mathrm{R}$ cuadrado ajustada $=$ $0,1213)$; *Estadísticamente significativo $\mathrm{p}<0,05$.

\section{DISCUSIÓN}

Los niveles de empatía global hallados son similares a los reportados por Alcorta $^{12}$ en México, WiIliams ${ }^{25}$ en Australia y Hojat ${ }^{26}$ en Estados Unidos; sin embargo estos podrían ser considerados altos al compararlos con los reportados por Saucedo ${ }^{27}$ en Perú, $\operatorname{Hasan}^{28}$ en Kuwait, Khademalhosseini ${ }^{6}$ en Irán, Shashikumar ${ }^{29}$ en la India y Alonso ${ }^{30}$ en Colombia. Esto sugiere que posiblemente varían dependiendo de otros factores entre los que podrían estar la cultura, el rol social, la carga académica y plan curricular.

En este estudio se exploró la relación entre los niveles de empatía con las características sociodemográficas, familiares y académicas, evidenciándose que las mujeres poseen mayores niveles de empatía. Estos resultados son similares a los reportados por Berg ${ }^{31}$ en Estados Unidos, Sánchez ${ }^{32}$ en Costa Rica y Nunes ${ }^{33}$ en Trinidad y Tobago, sugiriendo que el rol del género en la relación aprendiz-paciente puede ejercer una influencia basados en la forma de abordar las relaciones interpersonales. En este sentido, algunos autores han explicado que las mujeres pueden desarrollar un tipo diferente de atención con una mayor capacidad de identificarse con las experiencias del paciente y sus sentimientos $^{34,35}$. Contrario a esto, varios estudios en estudiantes de Ciencias de la Salud no encontraron estas diferencias ${ }^{15,36,37}$, por lo cual se sugiere que aún no está completamente claro si los niveles de empatía están influenciados por el sexo. De igual forma se podría pensar que estas diferencias pueden estar influidas por otros factores, algunos motivacionales relacionados con la etapa de desarrollo en que se encuentren y otros por el comportamiento individual ligado a la calidad humana de cada estudiante. Estos factores comienzan a destacarse desde el momento en que existe el primer contacto con el paciente, al sentirse muy comprometidos con el estado general de este y darle una mirada diferente desde la perspectiva humanística de sentir respeto por el otro ${ }^{38}$.

Al comparar los niveles de empatía global con las características familiares, se evidenció una relación significativa con la funcionalidad familiar, lo que constituye un hallazgo innovador, debido a que no se encontraron reportes que evaluaran directamente estas variables con la empatía en es- tudiantes de Medicina, sin embargo Madera ${ }^{39}$ reportó esta asociación en estudiantes de Odontología. Además, algunos estudios han evaluado otras características familiares en estudiantes de Medicina, como el realizado por Hasan ${ }^{28}$,quien evaluó los niveles de empatía y las características de los padres, encontrando que los niveles de empatía están relacionados con el nivel de educación de la madre y con la relación que tienen los estudiantes con su madre, concluyendo que los estudiantes que tienen y que tuvieron en la infancia una relación satisfactoria con sus madres poseen mayores niveles de empatía. Asimismo Hojat ${ }^{26}$ concluyó que los niveles de empatía en estudiantes de medicina son directamente proporcionales a los niveles de satisfacción con la relación de la madre, lo que demuestra la importancia de la familia y las relaciones entre sus miembros para el desarrollo de la capacidad de interacción con la sociedad. Esto se puede deber a que la familia tradicionalmente se ha concebido como una fuente que provee a los jóvenes de valores, creencias, costumbres, entre otros aspectos que ayudan a mantener su integridad física y emocional. Además otorga entrenamiento en la habilidad comunicativa cuando enfrenta a los jóvenes a relacionarse y resolver discrepancias con otros individuos que difieren en personalidad, edad, género y creencias ${ }^{40}$. Esta influencia de la familia funcional podría ser responsable de que los estudiantes de Medicina reproduzcan esta conducta cuando forjan la relación aprendiz-paciente, mostrando cierto grado de sensibilización y comprensión hacia la percepción o sentir de su paciente.

Los resultados sugieren relación entre los niveles de empatía con el rendimiento académico y promedio académico acumulado, en donde los que tienen mejor rendimiento académico poseen mayores niveles de empatía. Esto es similar a lo reportado por Castaño ${ }^{41}$ quien, en su estudio, describió el perfil psicosocial de los estudiantes de Medicina de la Universidad de Manizales en Colombia y su relación con el rendimiento académico y, utilizando los mismos instrumentos para medir y definir estas variables que en el presente estudio, concluyó que el rendimiento académico se relaciona con la empatía en sus tres dimensiones.

Por otra parte, se evidenció que los estudiantes que tenían la profesión de Medicina como primera 
opción de estudio reportaron mayores niveles de empatía, lo que demuestra la influencia que genera la elección de la profesión como primera opción de estudio sobre los niveles de empatía, pues se podría pensar que quien selecciona una profesión de primera intención es motivado por la afinidad y preferencia hacia la misma. Carrasco ${ }^{42}$ afirma que en la orientación vocacional existen otros factores para la toma de decisión como la personalidad, percepciones de autoeficacia y los propios intereses, de tal manera que si existe tal compromiso de los factores emocionales, conductuales y perceptivos del individuo en la selección de la profesión, se espera disponer con mayor razón de los mismos durante el desarrollo de la misma, convirtiéndose en habilidades y factores protectores para lograr el desempeño esperado. También es conveniente mencionar que autores como Toso ${ }^{43}$ han señalado que para los estudiantes de Ciencias de la Salud una motivación frecuente para seleccionar la profesión es el interés social o el interés por ayudar a los demás, ya que estas profesiones requieren de esta habilidad que algunos estudiantes creen poseer y que sin lugar a dudas constituye un elemento inherente a la relación empática.

Entre las limitaciones del presente estudio está el diseño transversal del mismo, el cual impide determinar si los niveles de empatía reportados han sido desarrollados durante los años de formación o si corresponden a una habilidad previa de los estudiantes; sin embargo la realización de procesos como la aleatorización, calibración, estandarización y la utilización de instrumentos validados permiten aportar resultados confiables; en este sentido el valor de confiabilidad de la EEMJ hallado es consistente con los valores reportados previamente $\mathrm{e}^{12,44,45}$, aportando evidencia para el uso de esta escala en la población objeto de estudio.

En conclusión la empatía es una cualidad importante para que un individuo logre ingresar a estudiar una profesión del área de la salud, donde se necesita la ayuda desinteresada para contribuir con la sanación del paciente; siendo igual de importante como equivalente a la inteligencia y a otras destrezas $^{46}$. Desde esta perspectiva y, teniendo en cuenta los hallazgos de este estudio, que posiblemente pueden reflejar el comportamiento de los futuros profesionales de la Salud debido a que la relación empática con sus pacientes podría depender de la formación integral que han recibido, es pertinente destacar la identificación de la familia como grupo de apoyo social, cuya influencia puede favorecer el desempeño de los estudiantes y futuros profesionales tras mejorar los niveles de empatía. Por tanto, se sugiere que algunas dependencias de la Universidad de Cartagena como bienestar universitario podrían reenfocar sus actividades para promover la inclusión y participación de familiares durante el proceso de formación educativa. Además, es posible contribuir a través del desarrollo de habilidades interpersonales en los educandos, incluyendo estrategias que permitan monitorear de manera continua el progreso del estudiante duran- te todo los años de formación para detectar de forma temprana cambios negativos en el rendimiento académico. En este sentido los estudiantes en estas condiciones, una vez identificados, podrían someterse a oportunas asesorías con trabajo social, salud familiar y psicología para determinar la causa de esta situación y en la medida posible contribuir a su resolución.

\section{AGRADECIMIENTOS}

A la Mg. Giselle Roxane Fetis Navarrete, profesora del Centro de Excelencia CIGES, Universidad de La Frontera (Temuco-Chile) por su invaluable apoyo y a los estudiantes de la Facultad de Medicina de la Universidad de Cartagena que participaron en este estudio.

\section{BIBLIOGRAFÍA}

1. Chen D, Lew R, Hershman W, Orlander J. A cross-sectional measurement of medical student empathy. J Gen Intern Med. 2007;22(10):1434-8.

2. Hojat M, Mangione S, Nasca TJ, et al. An empirical study of decline in empathy in medical school. Med Educ. 2004;38(9):934-41.

3. Glaser KM, Markham FW, Adler HM, McManus PR, Hojat M. Relationships between scores on the Jefferson Scale of physician empathy, patient perceptions of physician empathy, and humanistic approaches to patient care: a validity study. Med Sci Monit. 2007;13(7):291-4.

4. Wen D, Ma X, Li H, et al. Empathy in Chinese medical students: psychometric characteristics and differences by gender and year of medical education. BMC Med Educ. 2013;13:130.

5. Dehning S, Gasperi S, Krause D, et al. Emotional and cognitive empathy in first-year medical students. ISRN Psychiatry. 2013;2013:801530.

6. Khademalhosseini M, Khademalhosseini Z, Mahmoodian F. Comparison of empathy score among medical students in both basic and clinical levels. J Adv Med Educ Prof. 2014;2(2):88-91.

7. Leombruni $P$, Di Lillo $M$, Miniotti $M$, et al. Measurement properties and confirmatory factor analysis of the Jefferson Scale of Empathy in Italian medical students. Perspect Med Educ. 2014;3(6):419-30.

8. Pedersen R. Empirical research on empathy in medicineA critical review. Patient Educ Couns. 2009;76(3):307-22.

9. Hojat M, Mangione S, Nasca T. The Jefferson Scale of Physician Empathy: Development and Preliminary Psychometric Data. Educational and Psychological Measurement. 2001;61(2):349-65.

10. Babar M. An assessment of dental students' empathy levels in Malaysia. Int J Med Educ. 2013;4:223-29.

11. Shariat SV, Habibi M. Empathy in Iranian medical students: measurement model of the Jefferson scale of empathy. Med Teach. 2013;35(1):913-8.

12. Alcorta A, González J, Tavitas S, Rodríguez F, Hojat M. Validación de la escala de empatía médica de Jefferson en estudiantes de Medicina mexicanos. Salud Mental. 2005;28(5):57-63.

13. Silva M, Arboleda J, Díaz V. Orientación empática en estudiantes de Medicina en una universidad de República Dominicana. Educación Médica Superior. 2014;28:74-83.

14. Sandoval L, Reducindo R, Islas A. Funcionamiento familiar y empatía en los médicos residentes del Instituto de Seguridad y Servicios Sociales de los Trabajadores del Estado. Rev Esp Med Quir. 2011:16(4):221-28.

15. Erazo A, Alonso L, Rivera I, Zamorano A, Díaz V. Evaluación de la Orientación Empática en estudiantes de odontología de la Universidad Metropolitana de Barranquilla (Colombia). Salud Uninorte. 2012;28(3):354-63.

16. Smilkstein G. The family APGAR: a proposal for a family function test and its use by physicians. J Fam Pract. 1978;6(6):1231-9.

17. Forero L, Avendaño M, Duarte Z, Campo A. Consistencia interna y análisis de factores de la escala APGAR para eva- 
luar el funcionamiento familiar en estudiantes de básica secundaria. Rev Colomb Psiquiatr. 2006;35(1):23-29.

18. Smilkstein $\mathrm{G}$, Ashworth $\mathrm{C}$, Montano $\mathrm{D}$. Validity and reliability of the family APGAR as a test of family function. J Fam Pract. 1982;15(2):303-11.

19. Bellón J, Delgado A, Luna J, Lardelli P. Validez y fiabilidad del cuestionario de función familiar Apgar-familiar. Aten Primaria. 1996;18(6):289-95.

20. Rodriguez Fernandez E, Gea Serrano A, Gomez Moraga A, Garcia Gonzalez JM. [Apgar questionnaire in the study of family function]. Aten Primaria. 1996;17(5):338-41.

21. Hojat M, Gonnella JS, Nasca TJ, et al. Physician empathy: definition, components, measurement, and relationship to gender and specialty. Am J Psychiatry. 2002;159(9):15639 .

22. Davis M. Measuring individual differences in empathy: evidence for multidimensional approach. J Pers Soc Psychol. 1983;44:113-126.

23. Kunyk D, Olson JK. Clarification of conceptualizations of empathy. J Adv Nurs. 2001;35(3):317-25.

24. Fields SK, Hojat M, Gonnella JS, et al. Comparisons of nurses and physicians on an operational measure of empathy. Eval Health Prof. 2004;27(1):80-94.

25. Williams B, Brown T, McKenna L, et al. Empathy levels among health professional students: a cross-sectional study at two universities in Australia. Adv Med Educ Pract. 2014;5:107-13.

26. Hojat M, Zuckerman M, Magee M, et al. Empathy in medical students as related to specialty interest, personality, and perceptions of mother and father. Personality and Individual Differences. 2005;39(7):1205-15.

27. Saucedo L. Empatía en estudiantes de medicina humana de la Universidad Nacional Pedro Ruiz Gallo. Mayo- Junio del 2011. Rev. cuerpo méd. HNAAA. 2012;5(3):17-21.

28. Hasan S, Al-Sharqawi N, Dashti F, et al. Level of empathy among medical students in Kuwait University, Kuwait. Med Princ Pract. 2013;22(4):385-9.

29. Shashikumar R, Chaudhary R, Ryali VS, et al. Cross sectional assessment of empathy among undergraduates from a medical college. Med J Armed Forces India. 2014;70(2):179-85

30. Alonso L, Caro S, Erazo A, Díaz V. Evaluación de la orientación empática en estudiantes de medicina de la Universidad del Norte. Barranquilla (Colombia). Salud Uninorte. Barranquilla (Col.) 2013;29(1):22-33.

31. Berg K, Blatt B, Lopreiato J, et al. Standardized patient assessment of medical student empathy: ethnicity and gender effects in a multi-institutional study. Acad Med. 2015;90(1):105-11.

32. Sánchez J L, Padilla G M, Rivera U I, Zamorano A A, Díaz
N V. Niveles de orientación empática en los estudiantes de Odontología. Educación Médica Superior. 2013;27:216-25.

33. Nunes P, Williams S, Sa B, Stevenson K. A study of empathy decline in students from five health disciplines during their first year of training. Int J Med Educ. 2011;2:12-17.

34. Rivera I, Arratia R, Zamorano A, Díaz V. Evaluación del nivel de orientación empática en estudiantes de Odontología. Salud Uninorte. Barranquilla (Col.) 2011;27 (1):63-72.

35. Sherman JJ, Cramer A. Measurement of changes in empathy during dental school. J Dent Educ. 2005;69(3): 338-45.

36. Beattie A, Durham J, Harvey J, Steele J, McHanwell S. Does empathy change in first-year dental students? Eur J Dent Educ. 2012;16(1):e111-6.

37. Loureiro J. Empatia na relação médico-doente. Evolução em Alunos do Primeiro Ano de Medicina e Contribuição para a Validação da Escala Jefferson em Portugal. Acta Med Port. 2011;24(S2): 431-42

38. Girón M, Beviá B, Medina E, Simón M. Calidad de la relación médico paciente y resultados de los encuentros clínicos en atención primaria de Alicante: un estudio con grupos focales. Rev Esp Salud Pública. 2002;76:561-75.

39. Madera M, Fétis G, Tirado L. Empatía y factores relacionados en estudiantes de odontología de la Universidad de Cartagena. Rev Colomb Investig Odontol. 2014;5 (14) 2014;5(14):69-80.

40. Guadarrama R, Marquez O, Veytia M, León A. Funcionamiento familiar en Estudiantes de nivel superior. Revista Electrónica de Psicología Iztacala. 2011;14(2):179-92.

41. Castaño J, Florido J, Galvis J, et al. El perfil psicosocial de los estudiantes de medicina de la Universidad de Manizales (Colombia) y su relación con el rendimiento académico 2011. Arch Med (Manizales). 2012;12(1):62-72.

42. Carrasco $E$, Zúñiga $C$, Espinoza J. Elección de carrera en estudiantes de nivel socioeconómico bajo de universidades chilenas altamente selectivas. Calidad en la Educación. 2014;40:95-128.

43. Toso A, Ayala M, Brunner V, et al. Intereses y perspectiva sobre la carrera de medicina: un contraste entre estudiantes de medicina de primero y séptimo año. Rev Méd Chile. 2012;140(5):609-15.

44. Hojat M, Gonnella JS, Nasca TJ, et al. The Jefferson Scale of Physician Empathy: further psychometric data and differences by gender and specialty at item level. Acad Med. 2002;77(10 Suppl):S58-60.

45. Tavakol S, Dennick R, Tavakol M. Psychometric properties and confirmatory factor analysis of the Jefferson Scale of Physician Empathy. BMC Med Educ. 2011;11:54.

46. Nash DA. Ethics, empathy, and the education of dentists. J Dent Educ. 2010;74(6):567-78.

\section{ANEXO. CUESTIONARIO ESTRUCTURADO}

Fecha:

\section{Características Sociodemográficas}

1. Edad:

2. Sexo: $(\mathrm{F})(\mathrm{M})$

3. Estado civil: Soltero () Casado () Viudo () Unión libre () Separado ()

4. Estudia y trabaja: Si () No ()

5. Religión: Católico () Evangélico () Adventista () Otro ()

6. Procedencia: Rural () Urbana ()

7. Estrato socioeconómico 1_2_3_4_5_6_

\section{Características académicas}

8. Año de formación: I () II () III () IV () V()

9. ¿Medicina fue su primera opción de estudio?: Si () No ()

10. ¿Ha reprobado alguna asignatura?: Si () No ()

11. ¿Ha reprobado más de una vez la misma asignatura?: Si () No ()

12. ¿Ha estudiado previamente otra carrera?: Si () No ()

\section{Características familiares}

13. Tipo de familia: extensa () nuclear () nuclear ampliada () binuclear () monoparental () otra () 